\title{
The Random Walk Representation of Classical Spin Systems and Correlation Inequalities
}

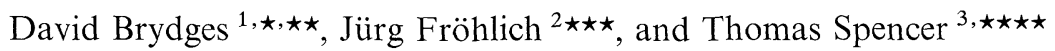 \\ 1 Departments of Mathematics and Physics, Indiana University, Bloomington, IN 47401, USA \\ 2 Institut des Hautes Etudes Scientifiques, 35 Route de Chartres, F-91440 Bures-sur-Yvette, France \\ 3 Courant Institute of Mathematical Sciences, 251 Mercer Street, New York, NY 10012, USA
}

\begin{abstract}
Ferromagnetic lattice spin systems can be expressed as gases of random walks interacting via a soft core repulsion. By using a mixed spinrandom walk representation we present a unified approach to many recently established correlation inequalities. As an application of these inequalities we obtain a simple proof of the mass gap for the $\lambda\left(\phi^{4}\right)_{2}$ quantum field model. We also establish new upper bounds on critical temperatures.
\end{abstract}

\section{Introduction}

In [1] Symanzik introduced a representation which expressed the $\phi^{4}$ quantum field model as a classical gas of Brownian paths which interact only when they cross. In $[2,3]$ and in this paper we have developed variants of this formalism which provide a transparent way to establish many inequalities.

In the first two sections we reconsider Symanzik's formalism. We prove two identities. The first identity expresses the spin system as a gas of random walks. In Sect. 3 we use this representation to obtain new upper bounds on critical temperatures. The second identity is a mixed spin-random walk representation. We combine this identity with chessboard estimates [4] and Griffiths [5] inequalities for $N=1$ or 2 component spins to obtain many new and useful results: In Sect. 4, we apply this formalism to show that for a class of classical spin models whose single spin distribution is monotone decreasing, there is always exponential decay of correlations. Hence there is no symmetry breaking. In Sect. 5 we give a new proof of the Lebowitz inequalities [6] and some generalizations related to Newman's Gaussian inequalities [7]. The following section rederives correlation inequalities recently found by Simon, Lieb and Rivasseau [8-10]. The final section of our paper is devoted to a new and elementary proof of the mass gap for the

$\star$ Partially supported by N.S.F. Grant No. 79-02490

$\star \star$ On leave from the University of Virginia

$\star \star \star$ Address after August 1982: Theor. Physics, ETH, Zürich, Switzerland

$\star \star \star \star$ Partially supported by N.S.F. Grant No. DMR 81-00417 
weakly coupled $\lambda\left(\phi^{4}\right)_{2}$ model. The proof uses only inequalities of Lieb-Rivasseau type and integration by parts.

We conclude this introduction by remarking that our results would extend to $N \geqq 3$ component models once the Griffiths inequalities are established. Some of our methods apply to lattice gauge theories. The first steps along these lines have been made in [3]. For abelian gauge groups, the techniques of [3] can be combined with the approach of the present paper to yield new correlation inequalities for lattice gauge theories. This, however, will not be developed in the present paper.

\section{Random Walks and Matrix Inverses}

The prototype for the expansions we are about to discuss is the following representation for the inverse of the finite difference Laplacian:

$$
\left[m^{2}-\Delta\right]_{i j}^{-1}=\sum_{\omega: i \rightarrow j}\left(\frac{1}{2 v+m^{2}}\right)^{|\omega|},
$$

where $\Delta$ denotes the finite difference Laplacian associated with functions on the lattice $\mathbb{Z}^{v},(v=1,2, \ldots) ; \omega$ is a nearest neighbor random walk of arbitrary length, $|\omega|$, on $\mathbb{Z}^{v}$, starting at $i \in \mathbb{Z}^{v}$, and ending at $j \in \mathbb{Z}^{v} ; m^{2}>0$.

The finite difference Laplacian, $\Delta$, is defined on functions on $\mathbb{Z}^{v}$ by

$$
\begin{array}{rlrl}
(\Delta f)_{i} & =\sum_{j} \Delta_{i j} f(j) \\
\Delta_{i j} & =-2 v & & \text { if } \quad i=j \\
& =1 & & \text { if } \quad i, j \quad \text { are nearest neighbors in } \mathbb{Z}^{v} \\
& =0 & & \text { otherwise. }
\end{array}
$$

The formula (1.1) is a standard result in the theory of random walks, however, in order to make this paper self-contained we will give the easy proof below.

The Laplacian is associated with nearest neighbor ferromagnetic interactions. We will give a more general expansion than (1.1) in order to be able to discuss systems with arbitrary ferromagnetic two-body interactions.

Let $J$ be a matrix such that

$$
\begin{array}{rlrl}
J_{i j}=J_{j i} \geqq 0 & \text { if } & i \neq j \\
& =0 & \text { if } & i=j ;
\end{array}
$$

$i, j$ are indices that run over a finite set, $L$, called the "lattice." Let $\Lambda$

$$
\Lambda \equiv\left(\lambda_{i} \delta_{i j}\right), \lambda_{i} \neq 0 \forall i
$$

be a diagonal matrix. The notation in the following lemma is described below it. 


\section{Lemma 1.1.}

$$
(\Lambda-J)_{i j}^{-1}=\sum_{\omega: i \rightarrow j}\left(\prod_{s \in \omega} J_{s}\right) \prod_{k \in L} \lambda_{k}^{-n(k, \omega)}
$$

If the right hand side converges absolutely, the matrix inverse exists and is given by (1.5).

Notation. $\omega$ is a random walk on $L$. This means $\omega$ is an ordered set of ordered pairs, called "steps" and denoted by $s$.

$$
\omega=\left\{\left(i_{1}, i_{2}\right),\left(i_{2}, i_{3}\right), \ldots,\left(i_{N-1}, i_{N}\right): i_{1}, \ldots, i_{N} \in L\right\},
$$

$n(k, \omega)$ is the number of times $\omega$ "hits" $k$. By definition, this is the number of elements in $\left\{i_{1}, i_{2}, \ldots, i_{N}\right\}$ which are equal to $k$. The "length" of $\omega,|\omega|$ is $N$ which is also equal to

$$
|\omega|=\sum_{k} n(k, \omega)
$$

By convention we shall consider a random walk of length one to be a single site (which is hit once). Such a random walk has no steps and an empty product in (1.5) is set equal to one. [These conventions allow (1.5) to be correct when $i=j$.]

$$
\omega: i \rightarrow j \Leftrightarrow i_{1}=i, \quad i_{N}=j .
$$

Remarks. (i) This lemma contains (1.1) as a special case. (ii) The expansion converges absolutely if for some $\xi>1$

$$
\left|\lambda_{i}\right| \geqq \xi \sum_{j} J_{i j}, \quad \forall i \in L .
$$

Proof of Lemma 1.1. Expand the left hand side of (1.5) in a Neumann series

$$
(\Lambda-J)^{-1}=\Lambda^{-1}+\Lambda^{-1} J \Lambda^{-1}+\Lambda^{-1} J \Lambda^{-1} J \Lambda^{-1}+\ldots
$$

The right hand side of (1.5) is a rewriting of this series. For example

$$
\begin{aligned}
\left(\Lambda^{-1} J \Lambda^{-1} J \Lambda^{-1}\right)_{i j} & =\sum_{\substack{i_{1}, i_{2}, i_{3} \in L \\
i_{1}=i, i_{3}=j}} \lambda_{i_{1}}^{-1} J_{i_{1} i_{2}} \lambda_{i_{2}}^{-1} J_{i_{2} i_{3}} \lambda_{i_{3}}^{-1} \\
& =\sum_{\omega}\left(\prod_{s \in \omega} J_{s}\right) \prod_{k \in L} \lambda_{k}^{-n(k, \omega)}
\end{aligned}
$$

where $\omega$ is summed over all two step random walks of the form

$$
\omega=\left\{\left(i_{1}, i_{2}\right),\left(i_{2}, i_{3}\right): i_{1}=i, i_{3}=j, i_{2} \in L\right\}
$$

End of proof.

We shall also need a formula, related to Lemma 1.1, for the determinant of $\Lambda-J$. Let $A$ be the set of random walks that begin and end at the same (arbitrary) point. We divide $A$ into equivalence classes by letting $\omega_{1}, \omega_{2} \in A$ be equivalent whenever $\omega_{1}, \omega_{2}$ have the same steps and the order of the steps in $\omega_{2}$ is a cyclic permutation of the order of the steps in $\omega_{1}$. We call the equivalence classes "random loops." Single points are not random loops. 
Given a random walk $\omega$ (or a random loop $\underset{\sim}{\omega}$ ) define

$$
J_{\omega} \equiv \prod_{s \in \omega} J_{s}
$$

\section{Lemma 1.2.}

$$
\operatorname{det}(\Lambda-J)^{-1}=\left(\prod_{i} \lambda_{i}\right)^{-1} \exp \left\{\sum_{\omega} J_{\omega} \prod_{i} \lambda_{i}^{-n(i, \omega)}\right\}
$$

$\underset{\sim}{\omega}$ is summed over all random loops. The sum converges absolutely if the entries of $\Lambda$ are sufficiently large in absolute value (see (1.9)).

Proof.

$$
\begin{aligned}
\operatorname{det}(\Lambda-J)^{-1} & =\operatorname{det} \Lambda^{-1} \operatorname{det}^{-1}\left(1-\Lambda^{-1} J\right) \\
& =\operatorname{det} \Lambda^{-1} \exp \left\{-\operatorname{tr} \log \left(1-\Lambda^{-1} J\right)\right\} \\
& =\operatorname{det} \Lambda^{-1} \exp \left\{\sum_{k=1}^{\infty} \frac{1}{k} \operatorname{tr}\left(\Lambda^{-1} J\right)^{k}\right\} \\
& =\operatorname{det} \Lambda^{-1} \exp \left\{\sum_{k=1}^{\infty} \frac{1}{k} \sum_{i \in L} \sum_{\substack{\omega: i \rightarrow i \\
|\omega|=k}}\left(\prod_{s \in \omega} J_{s}\right) \prod_{j \in L} \lambda_{j}^{-n(j, \omega)}\right\} .
\end{aligned}
$$

We have just used the idea in the proof of Lemma 1.1. We continue with:

$$
=\operatorname{det} \Lambda^{-1} \exp \left\{\sum_{\underset{\sim}{\omega}}\left(\prod_{s \in \underset{\sim}{\omega}} J_{s}\right) \prod_{j \in L} \lambda_{j}^{-n(j, \underset{\sim}{\omega})}\right\} .
$$

When the determinant of $\Lambda$ is written out explicitly we obtain Lemma 1.2.

\section{Symanzik's Polymer Representation and Integration by Parts with Random Walk}

The hamiltonian for our lattice spin system is

$$
H \equiv-\frac{1}{2} \sum_{i, j \in L} \sum_{\alpha=1, \ldots, N} S_{i}^{(\alpha)} J_{i j} S_{j}^{(\alpha)}
$$

$\alpha$ labels the components of our vector-valued spins $\mathbf{S}_{i}=\left(S_{i}^{(\alpha)}\right) . J$ is ferromagnetic. We impose the conditions:

$$
J_{i j}=J_{j i} \geqq 0 \quad \text { if } \quad i \neq j,=0 \quad \text { if } \quad i=j .
$$

The unnormalized expectation corresponding to our hamiltonian is given by

$$
[F] \equiv \int \prod_{i, \alpha} d S_{i}^{(\alpha)} g_{i}\left(\mathbf{S}_{i}^{2}\right) e^{-H} F,
$$

where $F$ (the "observable") is a function of the spins. The normalized expectation is

$$
\langle F\rangle \equiv[F] / Z,
$$

where $Z=[1]$ is the "partition function." The single spin distributions which are described by the smooth functions $g_{i}$ are assumed to fall off faster than 
exponentially, i.e.

$$
g_{i}(t) e^{c t} \rightarrow 0 \quad \text { as } \quad t \rightarrow \infty,
$$

for all $c$ and all $i$. We make this strong assumption solely to avoid uninteresting technical problems. Once we have achieved our estimates we shall relax it by taking suitable limits in those estimates.

The Polymer Representation. Following Symanzik, [1], we shall show that our lattice spin systems can be rewritten in terms of a gas of "random loops" or polymers. Our integration by parts formula is presented second because it is a mixture of this representation and the spin representation. However most of our results rely on the integration by parts formula so this section can be skipped if the reader wishes.

We substitute into the partition function, $Z$, according to

$$
g_{j}\left(\mathbf{S}_{j}^{2}\right)=\int_{\Gamma} \hat{g}_{j}\left(a_{j}\right) e^{-i a_{j} \mathbf{S}_{j}^{2}} d a_{j} .
$$

$\Gamma$ is the contour $\operatorname{Im} a=-\lambda$, where $\lambda$ is chosen sufficiently large, positive, that Lemmas $1.1,1.2$ will be applicable to $\lambda I-J . \hat{g}(a)$ is the analytic continuation of the Fourier transform of $g$. It exists by virtue of our assumption (2.5).

We obtain

$$
Z=\int_{\Gamma} \prod_{i} \hat{g}_{i}\left(a_{i}\right) d a_{i} \int \prod_{i} d \mathbf{S}_{i} \exp \left[-H-i \sum_{j} a_{j} \mathbf{S}_{j}^{2}\right]
$$

The $\mathbf{S}$ integrals are gaussian and can be evaluated:

$$
Z=\int_{\Gamma} \prod_{i} \hat{g}_{i}\left(a_{i}\right) d a_{i} \operatorname{det}^{-N / 2}[2 i a-J],
$$

where $2 i a-J$ is the matrix

$$
2 i a_{k} \delta_{k l}-J_{k l} ; \quad k, l \in L .
$$

We use Lemma 1.2 to represent the determinant:

$$
Z=\int_{\Gamma} \prod_{j} d a_{j} \hat{g}_{j}\left(a_{j}\right)\left(2 i a_{j}\right)^{-N / 2} \exp \left[\frac{N}{2} \sum_{\sim} J_{\omega} \prod_{l}\left(2 i a_{l}\right)^{-n(l, \omega)}\right] .
$$

We expand the exponential and write the result as

$$
Z=\sum_{n=0}^{\infty} \frac{1}{n !}\left(\frac{N}{2}\right)^{n} \sum_{\omega_{1}, \ldots, \omega_{n}}\left(\prod_{k=1}^{n} J_{\left(\mathcal{\nu}_{k} k\right.}\right) \exp \left[-U\left(\underset{\sim}{\omega_{1}}, \ldots, \underset{\sim}{\omega}\right)\right],
$$

where

$$
\begin{gathered}
\exp \left[-U\left({\underset{\sim}{1}}_{1}, \ldots,{\underset{\sim}{\omega}}_{n}\right)\right] \equiv \prod_{i} \int_{\Gamma} d a_{i} \hat{g}_{i}\left(a_{i}\right)\left(2 i a_{i}\right)^{-n\left(i, \omega_{1}, \ldots, \omega_{n}\right)}, \\
n\left(i,{\underset{\sim}{\omega}}_{1}, \ldots,{\underset{\sim}{\omega}}_{n}\right) \equiv n\left(i,{\underset{\sim}{\omega}}_{1}\right)+\ldots+n\left(i,{\underset{\sim}{\omega}}_{n}\right)+\frac{N}{2} .
\end{gathered}
$$


(2.11) displays the partition function for our lattice spin system as a partition function for a system of interacting random loops or "polymers." We show, below, that $\exp -U$ is real and positive.

We can repeat this derivation for $[P]$, an unnormalized expectation of a polynomial in the spins. The step analogous to the gaussian integration in going from (2.7) to (2.8) uses

$$
\begin{aligned}
& \int \prod_{i} d \mathbf{S}_{i} \exp \left(-\frac{1}{2} \sum_{i, j, \alpha} S_{i}^{(\alpha)} M_{i j} S_{j}^{(\alpha)}\right) P(\underset{\sim}{\mathbf{S}}) \\
& =\left.\operatorname{det}^{-N / 2}[M] \exp \left(\frac{1}{2} \sum_{i, j, \alpha} \frac{\partial}{\partial S_{i}^{(\alpha)}} M_{i j}^{-1} \frac{\partial}{\partial S_{j}^{(\alpha)}}\right) P\right|_{\mathbf{S}=0}
\end{aligned}
$$

where $M=2 i a-J$. The exponential of the differential operator is defined by its power series which is truncated when acting on a polynomial. We shall not prove this fairly well-known formula for the moments of a gaussian integral. By expanding $M^{-1}$ using Lemma 1.1 and continuing as before for $Z$ we obtain a polymer representation for $[P]$ and then, by dividing through by $Z$, for $\langle P\rangle$. To express the result we introduce:

$$
\begin{aligned}
Z\left(\omega_{1}, \omega_{2}, \ldots, \omega_{p}\right) \equiv & \sum_{n=0}^{\infty} \frac{1}{n !}\left(\frac{N}{2}\right)^{n} \sum_{\underset{\omega}{\omega_{1}}, \ldots, \omega_{n}}\left(\prod_{m=1}^{p} J_{\omega_{m}} \prod_{k=1}^{n} J_{\omega^{\prime} k}\right) \\
& \cdot \exp \left[-U\left(\underset{\sim}{\omega_{1}}, \ldots,{\underset{\sim}{n}}_{n}, \omega_{1}, \ldots, \omega_{p}\right)\right]
\end{aligned}
$$

for $p=1,2, \ldots$ [It is useful to note that $Z\left(\omega_{1}, \ldots, \omega_{p}\right) / Z$ is a correlation function for the polymer gas.]

Theorem 2.1 (Symanzik).

$$
\left\langle S_{i_{1}}^{(\alpha)} S_{i_{2}}^{(\alpha)} \ldots S_{i_{2 p}}^{(\alpha)}\right\rangle=\sum_{\omega_{1}, \ldots, \omega_{p}} Z\left(\omega_{1}, \ldots, \omega_{p}\right) / Z,
$$

$p=1,2, \ldots \omega_{1}, \ldots, \omega_{p}$ are summed over all random walks that begin and end at lattice sites in $\left\{i_{1}, \ldots, i_{2 p}\right\}$ in such a way that $\left\{i_{1}, \ldots, i_{2 p}\right\}$ is partitioned into disjoint pairs, one for each of $\omega_{1}, \ldots, \omega_{p}$.

Remark. exp $-U$ is real and positive because in (2.14) we can substitute

$$
(2 i a)^{-n}=\frac{1}{(n-1) !} \int_{0}^{\infty} t^{n-1} e^{-(2 i a) t} d t
$$

and find that

$$
\int_{\Gamma} d a \hat{g}(a)(2 i a)^{-n}=\int_{0}^{\infty} \frac{t^{n-1}}{(n-1) !} g(2 t) d t
$$

( $n$ can be fractional).

Integration by Parts. We are going to elaborate on the following well-known formula :

$$
\begin{aligned}
& \int \prod_{i} d S_{i} \exp \left(-\frac{1}{2} \sum_{i, j} S_{i} M_{i j} S_{j}\right) S_{k} F \\
& \quad=\int \prod_{i} d S_{i} \exp \left(-\frac{1}{2} \sum_{i, j} S_{i} M_{i j} S_{j}\right) \sum_{l} M_{k l}^{-1} \frac{\partial F}{\partial S_{l}} .
\end{aligned}
$$


(Until we reach the end of our discussion we will treat scalar spins for simplicity.) The real part of $M$ is positive definite. To obtain an analogue of this formula for our spin systems (which are nongaussian) we proceed as before by substituting into $\left[S_{i} F\right]$ the representation (2.6). If the a integrals are deferred, the $S$ integrals become gaussian and (2.17) can be applied with $M$ chosen to be the matrix

$$
M=2 i a-J \equiv\left(2 i a_{j} \delta_{j k}-J_{j k}\right) .
$$

We obtain

$$
\begin{aligned}
{\left[S_{i} F\right]=} & \int_{\Gamma} \prod_{j} d a_{j} \hat{g}_{j}\left(a_{j}\right) \int \prod_{j} d S_{j} e^{-H} \\
& \cdot \exp \left(-i \sum_{j} a_{j} S_{j}^{2}\right) \sum_{k}(2 i a-J)_{i k}^{-1} \frac{\partial F}{\partial S_{k}} .
\end{aligned}
$$

We expand $(2 i a-J)^{-1}$ using Lemma 1.1 and interchange the sum over $\omega$ with the $a, S$ integrals:

$$
\begin{gathered}
=\sum_{k} \sum_{\omega: i \rightarrow k} J_{\omega} \int_{\Gamma} \prod_{j} d a_{j} \hat{g}_{j}\left(a_{j}\right)\left(2 i a_{j}\right)^{-n(j, \omega)} \\
\cdot \int \prod_{j} d S_{j} e^{-H} \exp \left(-i \sum_{j} a_{j} S_{j}^{2}\right) \frac{\partial F}{\partial S_{k}} .
\end{gathered}
$$

Now we do the a integrals using

$$
\int d a \hat{g}(a)(2 i a)^{-n} e^{-i a S^{2}}=\int_{0}^{\infty} \frac{t^{n-1}}{(n-1) !} g\left(S^{2}+2 t\right) d t,
$$

which can easily be obtained with the help of (2.15), (2.16). We obtain

Theorem 2.2. Define measures $d v_{n}$ on $[0, \infty)$ by

$$
\begin{aligned}
d v_{n}(t) & \equiv \delta(t) d t \quad \text { if } \quad n=0 \\
& \equiv \frac{t^{n-1}}{(n-1) !} d t \quad \text { if } \quad n=1,2, \ldots
\end{aligned}
$$

To each random walk, $\omega$, on $L$ assign the product measure

$$
d v_{\omega}(t) \equiv \prod_{i \in L} d v_{n(i, \omega)}\left(t_{i}\right) .
$$

Then for any $i \in L, \alpha=1,2, \ldots, N$

$$
\left[S_{i}^{(\alpha)} F\right]=\sum_{j} \sum_{\omega: i \rightarrow j} J_{\omega} \int d v_{\omega}(t)\left[\frac{\partial F}{\partial S_{j}^{(\alpha)}}\right]_{t},
$$

where

$$
[(\cdot)]_{t} \equiv \int \prod_{i} g_{i}\left(\mathbf{S}_{i}^{2}+2 t_{i}\right) d \mathbf{S}_{i} e^{-H}(\cdot)
$$

and $F$ is any polynomial in the spins. 


\section{Estimates on the Critical Temperature}

We consider classical $N$ component rotators in the "infinite volume limit." Thus, we assume the lattice $L$ is a finite subset of an infinite translation invariant lattice, $L_{\infty}$, of points in $\mathbb{R}^{v}$. The simple cubic lattice $\mathbb{Z}^{v}$ is an example but other geometries are acceptable. We suppose the coupling matrix $J$ is translation invariant, i.e.,

$$
J=\left(J_{i j}\right)=\left(J_{|i-j|}\right)
$$

besides enjoying its usual properties, see (2.2). We set

$$
\begin{aligned}
H_{L} & \equiv-\frac{1}{2} \sum_{i, j \in L} \sum_{\alpha=1}^{N} S_{i}^{(\alpha)} J_{i j} S_{j}^{(\alpha)}, \\
{[(\cdot)]_{L} } & \equiv \int \prod_{i \in L, \alpha} d S_{i}^{(\alpha)} \delta\left(\mathbf{S}_{i}^{2}-1\right) e^{-H_{L}}(\cdot), \\
\langle(\cdot)\rangle_{L} & \equiv[(\cdot)]_{L} / Z_{L}, Z_{L} \equiv[1]_{L},
\end{aligned}
$$

and we define the (or : an) infinite volume limit by

$$
\langle F\rangle=\lim _{L \rtimes L \infty}\langle F\rangle_{L}
$$

where $F$ is an observable depending on finitely many spins. In (3.3) it may be necessary to pass to a subsequence to obtain existence of a limit. We shall shortly be presenting estimates which are uniform in $L$ which guarantee such compactness.

Define the coupling strength $\bar{J}$ by

$$
\bar{J} \equiv \sum_{j \in L_{\infty}} J_{i j}
$$

[By translation invariance $\bar{J}$ is independent of $i$.] By definition the critical coupling $\bar{J}_{c}$ is the supremum over values of $\bar{J}$ for which the two point function $\left\langle S_{i}^{(\alpha)} S_{j}^{(\alpha)}\right\rangle$ tends to zero, as $|i-j| \rightarrow \infty$.

The following theorem is an improvement of a mean field theory bound due to Simon and Aizenman and Simon $[8,11]$.

Theorem 3.1. (i) For an N-component classical rotator

$$
\bar{J}_{c} \geqq N \text {. }
$$

(ii) If the lattice is simple cubic in $v$ dimensions and $J_{i j}=\beta \geqq 0$ if $i$ and $j$ are nearest neighbors, zero otherwise, we can improve (3.5) to

$$
\beta_{c} \geqq \frac{N}{2 v}\left(1-\frac{1}{(N+2) v}\right)^{-1} .
$$

The first part of this theorem is due to Aizenman and Simon $[8,11]$. It says that the mean field theory prediction is a lower bound. We will present a proof of this theorem using Symanzik's representation. It should be pointed out that Symanzik's polymer representation is really just a good way of organizing the conventional expansion in terms of graphs and so we are really not doing anything very different from the graphical methods used by Simon and Aizenman, although our method potentially gives a somewhat stronger result; see (3.8), (3.9), and (3.12). 
Proof of Theorem 3.1. We shall show that if

$$
\bar{J} \equiv \sum_{j} J_{i j}<N
$$

then

$$
0 \leqq\left\langle S_{i}^{(\alpha)} S_{j}^{(\alpha)}\right\rangle_{L} \leqq \frac{1}{N}\left(1-N^{-1} J\right)_{i j}^{-1}
$$

uniformly in $i, j \in L$ and $L$, and the right side of (3.8) tends to 0 , as $|i-j| \rightarrow \infty$. More precisely, if (3.7) holds and

$$
\sum_{j}|i-j|^{2 m} J_{i j}<\infty
$$

for some $m=0,1,2, \ldots$, then

$$
\sum_{j}|i-j|^{2 m}\left\langle S_{i}^{(\alpha)} S_{j}^{(\alpha)}\right\rangle_{L} \leqq \text { const }<\infty,
$$

uniformly in $L$.

For, by (3.8) and the Fourier transformation

$$
0 \leqq\left\langle S_{i}^{(\alpha)} S_{j}^{(\alpha)}\right\rangle_{L} \leqq \frac{1}{N}\left(\frac{1}{2 \pi}\right)^{v} \int_{-\pi}^{\pi} \ldots \int_{-\pi}^{\pi} e^{i k \cdot(i-j)}\left(1-N^{-1} \hat{J}(k)\right)^{-1} d^{v} k,
$$

where

$$
\hat{J}(k)=\sum_{j} e^{-i k \cdot(i-j)} J_{i j}
$$

Since $J_{i j} \geqq 0$,

$$
\hat{J}(k) \leqq \hat{J}(0)=\bar{J}
$$

Thus, by (3.7),

$$
\left(1-N^{-1} \hat{J}(k)\right)^{-1}
$$

is a bounded, continuous function of $k$. This proves that

$$
\left(1-N^{-1} J\right)_{i j}^{-1}
$$

tends to 0 , as $|i-j| \rightarrow \infty$. If

$$
\sum_{j}|i-j|^{2 m} J_{i j}<\infty
$$

then $\hat{J}(k)$ is $2 m$ times continuously differentiable. The same is true for $\left(1-N^{-1} \hat{J}(k)\right)^{-1}$, provided (3.7) holds. Therefore (3.9) follows. Part (i) clearly follows from (3.8).

By Theorem 2.1 and a simple approximation argument,

$$
\left\langle S_{i}^{(\alpha)} S_{j}^{(\alpha)}\right\rangle_{L}=\sum_{\omega: i \rightarrow j} J_{\omega} Z^{-1} \sum_{n=0}^{\infty} \frac{1}{n !}\left(\frac{N}{2}\right)^{n} \sum_{\omega_{1}, \ldots, \omega_{n}}\left(\prod_{k=1}^{n} J_{()_{k}}\right) \exp \left[-U\left({\underset{\sim}{1}}_{1}, \ldots,{\underset{\sim}{\omega}}_{n}, \omega\right)\right] .
$$


Note that the right side of $(3.10)$ is manifestly positive, since $U\left({\underset{\sim}{\omega}}_{1}, \ldots,{\underset{\sim}{N}}_{n}, \omega\right)$ is real and $J_{\omega} \geqq 0$.

We calculate $\exp [-U]$ using $(2.12),(2.16)$ and find

$$
\begin{gathered}
\exp \left[-U\left({\underset{\sim}{1}}_{1}, \ldots,{\underset{\sim}{\omega}}_{n}, \omega\right)\right]=\prod_{i \in L} 2^{-n\left(i, \omega_{1}, \ldots, \omega_{n}\right)-n(i, \omega)} \\
\cdot\left[\left(n\left(i,{\underset{\sim}{1}}_{1}, \ldots,{\underset{\sim}{\omega}}_{n}\right)+n(i, \omega)-1\right) !\right]^{-1} .
\end{gathered}
$$

By comparing this with the corresponding evaluation of $\exp \left[-U\left({\underset{\sim}{\omega}}_{1}, \ldots, \underset{\sim}{\omega}\right)\right]-$ which is the same as (3.11) except that $n(i, \omega)$ is omitted - we find

$$
\begin{gathered}
\exp \left[-U\left({\underset{\sim}{\omega}}_{1}, \ldots,{\underset{\sim}{\omega}}_{n}, \omega\right)\right] \leqq \prod_{i \in \omega} 2^{-n(i, \omega)} \\
\cdot\left[\frac{N}{2}\left(\frac{N}{2}+1\right) \ldots\left(\frac{N}{2}+n(i, \omega)-1\right)\right]^{-1} \cdot \exp \left[-U\left({\underset{\sim}{\omega}}_{1}, \ldots,{\underset{\sim}{\omega}}_{n}\right)\right] .
\end{gathered}
$$

When this bound is substituted into the expression (2.14) for the two point function, the factor of $Z$ can be cancelled out [see (2.11)] yielding

$$
\left\langle S_{i}^{(\alpha)} S_{j}^{(\alpha)}\right\rangle_{L} \leqq \sum_{\omega: i \rightarrow j} J_{\omega} \prod_{k \in \omega} \frac{2^{-n(k, \omega)}}{\frac{N}{2}\left(\frac{N}{2}+1\right) \ldots\left(\frac{N}{2}+n(k, \omega)-1\right)} .
$$

Each factor is less than $N^{-n(k, \omega)}$ so (3.12) is less than

$$
\leqq \frac{1}{N} \sum_{\omega: i \rightarrow j} \prod_{s \in \omega}\left(\frac{1}{N} J_{s}\right)
$$

See (1.10).

If in (3.14) one drops the restriction that $\omega$ lie within $L$ (as opposed to $L_{\infty}$ ) one can resum the series over $\omega$ and obtains

$$
\leqq \frac{1}{N}\left(1-N^{-1} J\right)_{i j}^{-1}
$$

This completes the proof of (3.8) and of part (i).

To obtain part (ii) we return to (3.12) and substitute in our special form for $J$ :

$$
\begin{aligned}
\left\langle S_{i}^{(\alpha)} S_{j}^{(\alpha)}\right\rangle \leqq & \frac{1}{2} \sum_{m \geqq|i-j|}(\beta / 2)^{m} \sum_{\omega:\|\omega\| \|=m} \prod_{k \in \omega} \\
& \cdot\left[\frac{N}{2}\left(\frac{N}{2}+1\right) \ldots\left(\frac{N}{2}+n(k, \omega)-1\right)\right]^{-1},
\end{aligned}
$$

where $\|\omega\|$ is the number of steps of $\omega$. As in part (i) we enlarged the sum over random walks to all $\omega$ starting at $i$ of length greater than or equal to $|i-j|$. In addition $\omega$ is understood to be a nearest neighbor random walk. At each step $\omega$ has $2 v$ choices of nearest neighbors to hop to. For each of these possibilities the weight in the sum in (3.16) is less than or equal to $\beta N^{-1}$. We can do better than this by noting that if $\omega$ steps back to the site it just left, then according to (3.16) the weight is less than or equal to $(\beta / 2)(N / 2+1)^{-1}$ because then the site is visited twice 
and this is the factor associated by (3.16) to the second visit. Thus if

$$
\beta N^{-1}(2 v-1)+(\beta / 2)\left(\frac{N}{2}+1\right)^{-1} \equiv \alpha<1,
$$

we can dominate (3.16) by a geometric series and conclude exponential decay for the two point function. This means that the critical temperature $\beta_{c}$ obeys

$$
\beta_{c} N^{-1}(2 v-1)+\beta_{c}(N+2)^{-1} \geqq 1,
$$

and this is the same as (3.6). End of proof of theorem.

\section{Mass Generation in Spin Systems with Monotone Decreasing Single Spin Distributions}

In this section we combine the random walk expansion (Theorem 2.2) with reflection (or Osterwalder-Schrader) positivity, in the form of chessboard estimates and a spectral representation of the two spin correlation, in order to exhibit a mass gap in a class of spin systems with decreasing single spin distributions. The requirement of reflection positivity places strong restrictions on the two spin couplings, $J$, and the boundary conditions (b.c.) imposed on the system; see e.g. [4]. In order to avoid technicalities which would obscure the basic simplicity of our arguments, we only consider periodic b.c. and nearest neighbor couplings, although our results hold under rather more general hypotheses. (For an analysis of general two spin couplings, $J$, compatible with reflection positivity, see [4].) Thus, the lattice, $L$, is a simple cubic lattice wrapped on a torus,

$$
L=\mathbb{Z}_{2 N_{1}} \ldots \mathbb{Z}_{2 N_{v}},
$$

where $N_{1}, \ldots, N_{v}$ are finite integers, and

$$
J_{i j}= \begin{cases}1, & \text { if }|i-j|=1 \\ 0, & \text { otherwise }\end{cases}
$$

We only study the behavior of the two spin correlation, $\left\langle S_{i}^{(\alpha)} S_{j}^{(\alpha)}\right\rangle, \alpha=1, \ldots, N$, where $N=1,2,3, \ldots$ is the number of components of a classical spin, $\mathbf{S}$, but our methods have an obvious extension to higher spin correlations. by

The Hamilton function of the spin systems considered in this section is given

$$
\begin{aligned}
H^{(\varepsilon)} & \equiv-\frac{1}{2} \sum_{\substack{\alpha=1, \ldots, N \\
i, j \in L}} S_{i}^{(\alpha)}\left(\Delta_{i j}-\varepsilon \delta_{i j}\right) S_{j}^{(\alpha)} \\
& \equiv-\frac{1}{2}\left(\mathbf{S},\left(\Delta_{L}-\varepsilon\right) \mathbf{S}\right),
\end{aligned}
$$

where

$$
\Delta_{i j} \equiv J_{i j}-2 v \delta_{i j}, \quad \text { and } \quad \varepsilon>0 .
$$

Clearly, $\Delta \equiv \Delta_{L}$ is the finite difference Laplacian with periodic b.c., and the term proportional to $\varepsilon$ serves as an infrared (long distance) regulator which is to be 
removed at the end of our subsequent estimates, (i.e. $\varepsilon \rightarrow 0$ ). The unnormalized expectation of our system in finite volume is defined by

$$
[-]_{L}^{(\varepsilon)} \equiv \int-e^{-\beta H^{(\varepsilon)}} \prod_{i \in L} g\left(\mathbf{S}_{i}^{2}\right) d \mathbf{S}_{i},
$$

where $d \mathbf{S}_{i}$ is the Lebesgue measure on $\mathbb{R}^{N}$, and $g$ is a monotone decreasing function on $[0, \infty)$. The equilibrium expectation at inverse temperature $\beta$ for the system confined to $L$ is given by

$$
\langle-\rangle_{L}^{(\varepsilon)} \equiv[-]_{L}^{(\varepsilon)} / Z_{L}^{(\varepsilon)},
$$

where $Z_{L}^{(\varepsilon)}$ is the partition function, and

$$
\langle-\rangle \equiv \lim _{\varepsilon>0} \lim _{L>\mathbb{Z}^{v}}\langle-\rangle_{L}^{(\varepsilon)}
$$

is the equilibrium expectation in the thermodynamic limit, and with the infrared regulator removed. The quantities $[-]_{L, t}^{(\varepsilon)},\langle-\rangle_{L, t}^{(\varepsilon)}$, and $Z_{L, t}^{(\varepsilon)}$ are defined in the same way, but with $g\left(\mathbf{S}_{i}^{2}\right)$ replaced by $g\left(\mathbf{S}_{i}^{2}+2 t_{i}\right), 0 \leqq t_{i}<\infty$, for all $i$.

For couplings, $J$, as in (4.2), periodic b.c. at the boundary of $L$, see (4.1), and $H^{(\varepsilon)}$ given by (4.3), reflection positivity holds, and consequently one obtains the following chessboard estimate (see [12]):

$$
Z_{L, t}^{(\varepsilon)} \leqq \prod_{m \in L}\left[Z_{L, t \sim}^{(\varepsilon)} \equiv t_{m}\right]^{1 /|L|}
$$

with $|L| \equiv\left(2 N_{1}\right) \ldots\left(2 N_{v}\right)$. We define

$$
z_{L}^{(\varepsilon)}(t) \equiv\left[Z_{L, t, t}^{(\varepsilon)} / Z_{L}^{(\varepsilon)}\right]^{1 /|L|} .
$$

Then (4.8) yields

\section{Lemma 4.1.}

$$
Z_{L, \mathfrak{d}}^{(\varepsilon)} / Z_{L}^{(\varepsilon)} \leqq \prod_{m \in L} z_{L}^{(\varepsilon)}\left(t_{m}\right)
$$

We will now pause to outline the idea underlying this section. It is to combine Lemma 4.1 with

$$
\begin{aligned}
\left\langle S_{i}^{(\alpha)} S_{j}^{(\alpha)}\right\rangle_{L}^{(\varepsilon)}= & \frac{1}{\beta} \sum_{\omega: i \rightarrow j}\left(\prod_{k \in L} \beta^{n(k, \omega)}\right) \int d v_{\omega}(t) \\
& \cdot \prod_{k \in L} e^{-(2 v+\varepsilon) \beta t} Z_{L, t}^{(\varepsilon)} / Z_{L}^{(\varepsilon)}
\end{aligned}
$$

which comes from Theorem 2.2. We bound the ratio of partition functions on the right hand side using Lemma 4.1, whereupon the $t$ integrals become independent. [See the definition of $d v_{\omega}(t)$ in Theorem 2.2.] Each $t$ integral has the form either:

$$
\int_{0}^{\infty} \frac{t^{n-1}}{(n-1) !} e^{-(2 v+\varepsilon) \beta t} z_{L}^{(\varepsilon)}(t) d t, \quad \text { with } \quad n \geqq 1,
$$

or:

$$
\int_{0}^{\infty} \delta(t) e^{-(2 v+\varepsilon) \beta t} z_{L}^{(\varepsilon)}(t) d t \quad(n=0)
$$


depending on whether the random walk hits the site $n \geqq 1$ times or no $(n=0)$ times.

If the single spin distribution $g\left(\mathbf{S}_{i}^{2}\right)$ is monotone decreasing, then

$$
z_{L}^{(\varepsilon)}(t) \leqq 1,
$$

and by a straightforward calculation each $t$ integral is less than or equal to $1 /(2 v \beta)$. If we impose a slightly stronger condition than monotonicity on $g\left(\mathbf{S}^{2}\right)$, then we can improve this to

$$
\lim _{\varepsilon>0} \lim _{L \rtimes \mathbb{Z}^{\nu}} z_{L}^{(\varepsilon)}(t)<1,
$$

and then each $t$ integral with $n \neq 0$ is less than or equal to $\xi /(2 v \beta)$ with $\xi<1$. By combining the representation for the two point function with Lemma 4.1 and this estimate we obtain

$$
\left\langle S_{i}^{(\alpha)} S_{j}^{(\alpha)}\right\rangle \leqq\left(\frac{1}{\beta} \sum_{\omega: i \rightarrow j} \prod_{k \in L}\left(\frac{1}{2 v}\right)^{n(k, \omega)}\right) \xi^{|i-j|},
$$

using the fact that in getting from $i$ to $j$ the random walk $\omega$ must hit at least $|i-j|$ sites giving at least $|i-j|$ factors of $\xi$. The quantity in round brackets is, by (1.1), the matrix inverse of $-\Delta$, times $\beta^{-1}$.

This bound is actually divergent if the lattice is 2 or less dimensional because the inverse of $-\Delta$ does not exist. If the lattice is more than 2-dimensional it exhibits exponential decay of the two point function.

We will now go through this argument in more detail. Our main result is Theorem 4.4. We will show by means of a spectral representation of the two point function that the difficulty in two or less dimensions, alluded to above, can be circumvented. This is the purpose of Lemmas 4.2, 4.3, given below.

We now turn to the spectral representation of the two-spin correlation: We single out one axis of the lattice $L$, e.g. the $v$-axis. Vectors in a plane perpendicular to that axis are denoted by $\mathbf{j}, \mathbf{k}$, etc. Let $\langle-\rangle^{(\varepsilon)}$ be a thermodynamic limit of the states $\langle-\rangle_{L}^{(\varepsilon)}$. Clearly $\langle-\rangle^{(\varepsilon)}$ is translation invariant. We may thus define the partial Fourier transform

$$
\left\langle\tilde{S}^{(\alpha)}(\mathbf{k}, 0) \overline{\tilde{S}^{(\alpha)}(\mathbf{k}, t)}\right\rangle^{(\varepsilon)} \equiv \sum_{j} e^{i \mathbf{k}(\mathbf{j}-\mathbf{m})}\left\langle S_{(\mathbf{j}, 0)}^{(\alpha)} S_{(\mathbf{m}, t)}^{(\alpha)}\right\rangle^{(\varepsilon)} .
$$

Lemma 4.2 [4]. If $\left\langle S_{i}^{(\alpha)} S_{j}^{(\alpha)}\right\rangle^{(\varepsilon)}$ tends to 0 , as $|i-j| \rightarrow \infty$ then

$$
\left\langle\tilde{S}^{(\alpha)}(\mathbf{k}, 0) \overline{\tilde{S}^{(\alpha)}(\mathbf{k}, t)}\right\rangle^{(\varepsilon)}=\int_{(\lambda)} d \varrho(\lambda, \mathbf{k}) \lambda^{|t|-1},
$$

where $\mathrm{d} \varrho(\cdot, \mathbf{k})$ is a positive measure, for all $\mathbf{k},-\pi \leqq k_{\mu} \leqq \pi, \mu=1, \ldots, v-1$, and

$$
\operatorname{supp} d \varrho(\cdot, \mathbf{k}) \cong[-1,1] \text {. }
$$

Moreover

$$
\int_{(\lambda)} d \varrho(\lambda, \mathbf{k}) \frac{1-\lambda}{\lambda} \leqq 4 / \beta .
$$


Remarks. 1. Representation (4.10) is a fairly straightforward consequence of reflection positivity and the spectral theorem; see [4]. For the couplings $J$ defined in (4.2),

$$
\operatorname{supp} d \varrho(\cdot, \mathbf{k}) \subseteq[0,1]
$$

which follows from the positivity of the transfer matrix of these spin systems. By Fourier transformation in $t$ we obtain from (4.10)

$$
\left\langle\left|\hat{S}^{(\alpha)}(k)\right|^{2}\right\rangle^{(\varepsilon)}=\int d \varrho(\lambda, \mathbf{k}) \frac{1-\lambda^{2}}{\lambda\left(1+\lambda^{2}-2 \lambda \cos k_{v}\right)},
$$

with $k=\left(\mathbf{k}, k_{v}\right)$. The infrared bound proven in [13] guarantees that

$$
\left(2-2 \cos k_{v}\right)\left\langle\left|\hat{S}^{(\alpha)}(k)\right|^{2}\right\rangle^{(\varepsilon)} \leqq \beta^{-1} .
$$

The upper bound (4.12) then follows from (4.14), (4.15), and (4.13) by noticing that

$$
\max _{-\pi \leqq k_{v} \leqq \pi}\left(2-2 \cos k_{v} /\left(1+\lambda^{2}-2 \lambda \cos k_{v}\right)\right) \leqq 4(1+\lambda)^{-2} .
$$

2. We define the inverse correlation length (mass gap) $m(\beta, \varepsilon)$ by

$$
\begin{aligned}
m(\beta, \varepsilon) & \equiv \lim _{t \rightarrow \infty}-\frac{1}{t} \ln \left\langle S_{(0,0)}^{(\alpha)} S_{(0, t)}^{(\alpha)}\right\rangle^{(\varepsilon)} \\
& =\lim _{t \rightarrow \infty}-\frac{1}{t} \ln \int_{(\mathbf{k})} \int_{(\lambda)} d \varrho(\lambda, \mathbf{k}) \lambda^{|t|-1} .
\end{aligned}
$$

Suppose now that for some $m(\beta)$

$$
m(\beta, \varepsilon) \geqq m(\beta)>0
$$

for all $\varepsilon>0$. Then we claim that

$$
\operatorname{supp} d \varrho(\cdot, \mathbf{k}) \cong\left[0, e^{-m(\beta)}\right],
$$

for almost all k. This follows directly from (4.10) and (4.13). Thus, using (4.12),

$$
\int_{(\lambda)} \lambda^{-1} d \varrho(\lambda, \mathbf{k}) \leqq 4 / \beta\left(1-e^{-m(\beta)}\right)
$$

and, by Lemma 4.2 ,

$$
\begin{aligned}
\left\langle S_{(\mathbf{j}, 0)}^{(\alpha)} S_{(\mathbf{m}, t)}^{(\alpha)}\right\rangle^{(\varepsilon)} & \leqq\left\langle S_{(0,0)}^{(\alpha)} S_{(0, t)}^{(\alpha)}\right\rangle^{(\varepsilon)} \\
& \leqq 4 e^{-m(\beta) t} / \beta\left(1-e^{-m(\beta)}\right)
\end{aligned}
$$

uniformly in $\varepsilon$. By a suitable choice of the $v$-axis, $|t| \geqq \frac{1}{\sqrt{v}} \operatorname{dist}((\mathbf{j}, 0),(\mathbf{m}, t))$. Therefore we obtain from (4.18) by taking $\varepsilon \rightarrow 0$

$$
\left\langle S_{i}^{(\alpha)} S_{j}^{(\alpha)}\right\rangle \leqq \frac{4}{\beta\left(1-e^{-m(\beta)}\right)} e^{-(m(\beta) / \sqrt{v})|i-j|}
$$


Lemma 4.3. If for all $\varepsilon>0$ and for constants $K(\varepsilon)<\infty$ and $z<1, z$ independent of $\varepsilon$,

$$
\left\langle S_{i}^{(\alpha)} S_{j}^{(\alpha)}\right\rangle_{L}^{(\varepsilon)} \leqq K(\varepsilon) z^{|i-j|},
$$

provided $N_{1}, \ldots, N_{v}$ are sufficiently large (depending on $i, j$, and $\varepsilon$ ) then inequalities (4.18) and (4.19) hold.

Proof. Under the hypotheses of Lemma 4.3, in particular (4.20), we find

$$
m(\beta, \varepsilon) \equiv \lim _{t \rightarrow \infty}-\frac{1}{t} \ln \left\langle S_{(0,0)}^{(\alpha)} S_{(0, t)}^{(\alpha)}\right\rangle^{(\varepsilon)} \geqq \ln (1 / z)>0,
$$

uniformly in $\varepsilon$. This being (4.17), we are done.

We are now prepared for the first main result of this section.

Theorem 4.4. If for $\varepsilon>0$ sufficiently small and $L$ large enough, $z_{L}^{(\varepsilon)}(t)$ (defined in (4.9)) is monotone decreasing in $t \in[0, \infty)$, and

$$
z_{L}^{(\varepsilon)}(t) \leqq z_{0}<1,
$$

for all $t \geqq t_{0}$, for some finite $t_{0}$, then

$$
\left\langle S_{i}^{(\alpha)} S_{j}^{(\alpha)}\right\rangle \leqq c_{1} e^{-c_{2}|i-j|},
$$

in particular there is no long range order and no symmetry breaking in the two spin correlation.

Remarks. 1. When $N=1,(4.21)$ implies that all connected correlations fall off exponentially, with decay rate $\geqq c_{2}$. This follows by using FKG inequalities, [14].

2. Let

$$
A=\left(\alpha_{1}, \ldots, \alpha_{n}\right), X=\left(j_{1}, \ldots, j_{n}\right), \quad \text { and } \quad S_{X}^{A}=\prod_{m=1}^{n} S_{j_{m}}^{\left(\alpha_{m}\right)} .
$$

Let $X+a=\left(j_{1}+a, \ldots, j_{n}+a\right)$. Suppose now that there exists some $\alpha \in\{1, \ldots, N\}$ which occurs an odd number of times in $A$ and $B$. Then, under the hypotheses of Theorem 4.1,

$$
\left\langle S_{X}^{A} S_{Y+a}^{B}\right\rangle \leqq c(X, Y) e^{-c_{2} a} .
$$

The proof of (4.22) is a straightforward extension of our proof of (4.21) which is given below.

3. We shall show that the hypotheses of Theorem 4.1 are true if, for example,

$$
g\left(\mathbf{S}^{2}\right)= \begin{cases}1, & |\mathbf{S}| \leqq \mathscr{R} \\ 0, & |\mathbf{S}|>\mathscr{R} .\end{cases}
$$

For $N=1$, this result is already contained in [15].

(b) $g\left(\mathbf{S}^{2}\right)$ is strictly monotone decreasing, and

$$
\int g\left(\mathbf{S}^{2}\right)|\mathbf{S}|^{n} d \mathbf{S}<\infty,
$$

for some $\eta>0$; e.g. $g\left(\mathbf{S}^{2}\right)=\left(1+|\mathbf{S}|^{2}\right)^{-\alpha}$, with $\alpha>N$, (when $v \geqq 3$, strict monotonicity suffices). 
(c) $v \geqq 3 ; g$ monotone decreasing, with

$$
0<g_{\infty} \equiv \lim _{|\mathbf{S}| \rightarrow \infty} g\left(\mathbf{S}^{2}\right)<g(0) \equiv g_{0}<\infty .
$$

These results are essentially best possible, because if $g$ were positive and constant the model is a massless Gaussian whose spin-spin correlation does not have exponential decay.

Proof of Theorem 4.4. If we combine the random walk expansion (Theorem 2.2) with the chessboard estimate (Lemma 4.1) we obtain the following upper bound on the two spin correlation

$$
\left\langle S_{i}^{(\alpha)} S_{j}^{(\alpha)}\right\rangle \leqq \frac{1}{\beta} \sum_{\omega: i \rightarrow j} \prod_{k \in L} \beta^{n(k, \omega)} z_{L}^{(\varepsilon)}(n(k, \omega)),
$$

where

$$
z_{L}^{(\varepsilon)}(n) \equiv \int_{0}^{\infty} \frac{t^{n-1}}{(n-1) !} e^{-(2 v+\varepsilon) \beta t} z_{L}^{(\varepsilon)}(t) d t .
$$

The factor $\exp [-(2 v+\varepsilon) \beta t]$ has appeared, because the diagonal part of the Hamiltonian $H^{(\varepsilon)}$, see (4.3), must be treated as one factor of the single spin distribution in Theorem 2.2. We now claim that

$$
(2 v+\varepsilon)^{n} \beta^{n} z_{L}^{(\varepsilon)}(n) \equiv p_{L}^{(\varepsilon)}(n)
$$

is monotone decreasing in $n$, and

$$
p_{L}^{(\varepsilon)}(1) \leqq z<1,
$$

if $\varepsilon$ is so small and $L$ so large that the hypotheses of Theorem 4.4 hold. In this case

$$
\begin{aligned}
\left\langle S_{i}^{(\alpha)} S_{j}^{(\alpha)}\right\rangle_{L}^{(\varepsilon)} \leqq & \frac{1}{\beta} \sum_{\omega: i \rightarrow j} \prod_{k \in L}(2 v+\varepsilon)^{-n(k, \omega)} \\
& \cdot\left(p_{L}^{(\varepsilon)}(1)\right)^{|i-j|} .
\end{aligned}
$$

We have used the fact that each $\omega$ starting at $i$ and ending at $j$ must visit at least $|i-j|$ different lattice sites at least once which by $(4.24 \mathrm{a})$ and $(4.24 \mathrm{~b})$ yields the factor $\left(p_{L}^{(\varepsilon)}(1)\right)^{\{i-j\}}$. By Lemma 1

$$
\sum_{\omega: i \rightarrow j} \prod_{k}(2 v+\varepsilon)^{-n(k, \omega)}=\left(\varepsilon-\Delta_{L}\right)_{i j}^{-1}
$$

where $\Delta_{L}$ is the finite difference Laplacian with periodic b.c. at the boundary of $L$. Thus, using (4.24a) and (4.24b)

$$
\left\langle S_{i}^{(\alpha)} S_{j}^{(\alpha)}\right\rangle_{L}^{(\varepsilon)} \leqq K(\varepsilon) z^{|i-j|}
$$

with $K(\varepsilon) \equiv \sup _{i, j} \frac{1}{\beta}\left(\varepsilon-\Delta_{L}\right)_{i j}^{-1}$, and $z<1$. By Lemma 4.3,

$$
\lim _{L \rightarrow \mathbb{Z}^{v}}\left\langle S_{i}^{(\alpha)} S_{j}^{(\alpha)}\right\rangle_{L}^{(\varepsilon)} \leqq \frac{4}{\beta\left(1-e^{-m(\beta)}\right)} e^{-(m(\beta) / \sqrt{v})|i-j|},
$$


with $m(\beta) \equiv \ln (1 / z)>0$. This reduces the proof of Theorem 4.1 to the Proof of (4.24). By rescaling the $t$ variable we see that

$$
p_{L}^{(\varepsilon)}(n)=\int_{0}^{\infty} \frac{t^{n-1}}{(n-1) !} e^{-t} \tilde{z}(t) d t,
$$

with $\tilde{z}(t)=z_{L}^{(\varepsilon)}(t / \beta(2 v+\varepsilon))$. The measure

$$
d P_{n}(t) \equiv \frac{t^{n-1}}{(n-1) !} e^{-t} d t
$$

is a probability measure. By hypothesis, $z_{L}^{(\varepsilon)}(t)$ is monotone decreasing, and $z_{L}^{(\varepsilon)}(0)=1$. Thus $\tilde{z}(t)$ is monotone decreasing and bounded above by 1 . We now extend the definition of $d P_{n}(t)$ to arbitrary real values of $n \geqq 1$. In order to prove the first part of (4.24a) and (4.24b) it then suffices to show that

$$
\begin{aligned}
\frac{d}{d n} \int d P_{n}(t) \tilde{z}(t) & =\int d P_{n}(t) \log t \tilde{z}(t)-\int d P_{n}(t) \log t \int d P_{n}(t) \tilde{z}(t) \\
& \leqq 0 .
\end{aligned}
$$

The left side can be rewritten as

$$
\int d P_{n}(t) d P_{n}\left(t^{\prime}\right)\left[\log t-\log t^{\prime}\right]\left[\tilde{z}(t)-\tilde{z}\left(t^{\prime}\right)\right]
$$

which is negative, since $\log t$ is monotone increasing, and $\tilde{z}(t)$ is monotone decreasing. Finally, the inequality

$$
p_{L}^{(\varepsilon)}(1)=\int_{0}^{\infty} e^{-t} \tilde{z}(t) d t \leqq z<1
$$

follows immediately from the definition of $\tilde{z}(t)$ and the hypotheses of Theorem 4.1, provided $\varepsilon$ is small enough and $L$ large enough. This completes our proof of (4.24a) and (4.24b) and of Theorem 4.1.

Remarks. If $g$ is a monotone decreasing function on $[0, \infty)$ then

with

$$
z_{L}^{(\varepsilon)}(t) \equiv\left[Z_{L, t}^{(\varepsilon)} / Z_{L}^{(\varepsilon)}\right]^{1 /|L|},
$$

$$
Z_{L, t}^{(\varepsilon)} \equiv \int e^{-\beta H^{(\varepsilon)}} \prod_{j \in L} g\left(\mathbf{S}_{j}^{2}+2 t\right) d \mathbf{S}_{j},
$$

is clearly monotone decreasing in $t$. In order to find examples of single spin distributions, $g$, for which the hypotheses of Theorem 4.1 are true, it therefore suffices to choose $g$ to be monotone decreasing and then show that, for $\varepsilon$ small and $L$ large

$$
z_{L}^{(\varepsilon)}(t) \leqq z_{0}<1, \quad t \geqq t_{0},
$$

for some $t_{0}<\infty$.

Examples. (a)

$$
g\left(\mathbf{S}^{2}\right)= \begin{cases}1, & |\mathbf{S}|^{2}<\mathscr{R}, \\ 0, & \text { otherwise }\end{cases}
$$


In this example

$$
\begin{aligned}
Z_{L, t}^{(\varepsilon)} & =\int e^{\frac{\beta}{2}\left(\mathbf{S},\left(\Delta_{L}-\varepsilon\right) \mathbf{S}\right)} \prod_{j \in L} g\left(\mathbf{S}_{j}^{2}+2 t\right) d \mathbf{S}_{j} \\
& =(1-2 t / \mathscr{R})^{N|L| / 2} \int \exp \left[\frac{\beta}{2}(1-2 t / \mathscr{R})\left(\mathbf{S},\left(\Delta_{L}-\varepsilon\right) \mathbf{S}\right)\right] \prod_{j \in L} g\left(\mathbf{S}_{j}^{2}\right) d \mathbf{S}_{j} \\
& \equiv(1-2 t / \mathscr{R})^{N|L| / 2} Z^{\prime}(t) .
\end{aligned}
$$

Thus

$$
\begin{aligned}
\frac{\partial}{\partial t} \log z_{L}^{(\varepsilon)}(t) & =\frac{\partial}{\partial t} \frac{1}{|L|} \log Z_{L, t}^{(\varepsilon)} \\
& =-\frac{N}{\mathscr{R}}(1-2 t / \mathscr{R})^{-1}+\frac{\beta}{\mathscr{R}}\left\langle\frac{1}{|L|}\left(\mathbf{S},\left(-\Delta_{L}+\varepsilon\right) \mathbf{S}\right)\right\rangle_{L}^{(\varepsilon)}(t)
\end{aligned}
$$

where $\langle-\rangle_{L}^{(\varepsilon)}(t)$ is the expectation $\langle-\rangle_{L}^{(\varepsilon)}$, with $\beta$ replaced by $\beta(1-2 t / \mathscr{R})$. By the infrared bound [13]

$$
-\left\langle\frac{1}{|L|}\left(\mathbf{S}, \Delta_{L} \mathbf{S}\right)\right\rangle_{L}^{(\varepsilon)}(t) \leqq \frac{N}{\beta(1-2 t / \mathscr{R})} .
$$

Since $\operatorname{supp} g=\{\mathbf{S}:|\mathbf{S}| \leqq \mathscr{R}\}$,

$$
\varepsilon\left\langle\left|\mathbf{S}_{0}\right|^{2}\right\rangle_{L}^{(\varepsilon)}(t) \leqq \varepsilon \mathscr{R}^{2}
$$

and

$$
\left\langle\frac{1}{|L|}\left(\mathbf{S},-\Delta_{L} \mathbf{S}\right)\right\rangle_{L}^{(\varepsilon)}(t) \leqq 4 v \mathscr{R}^{2} .
$$

Thus

$$
\begin{aligned}
& \left\langle\frac{1}{|L|}\left(\mathbf{S},\left(-\Delta_{L}+\varepsilon\right) \mathbf{S}\right)\right\rangle_{L}^{(\varepsilon)}(t) \\
& \quad \leqq \min \left(\frac{N}{\beta(1-2 t / \mathscr{R})}, 4 v \mathscr{R}^{2}\right)+\varepsilon \mathscr{R}^{2},
\end{aligned}
$$

hence, using (4.29),

$$
\begin{gathered}
\frac{\partial}{\partial t} \log z_{L}^{(\varepsilon)}(t) \leqq-\frac{N}{\mathscr{R}} \frac{1}{1-2 t / \mathscr{R}}+\beta \varepsilon \mathscr{R} \\
+\min \left(\frac{N}{\mathscr{R}} \frac{1}{1-2 t / \mathscr{R}}, 4 \beta \nu \mathscr{R}\right) .
\end{gathered}
$$

Thus, for all $0<\varepsilon \leqq 1, \mathscr{R}<\infty$, there exists some $t_{1}<\mathscr{R} / 2$ such that

$$
\frac{\partial}{\partial t} \log z_{L}^{(\varepsilon)}(t)<0
$$

for $t>t_{1}$, with upper bounds on $\frac{\partial}{\partial t} \log z_{L}^{(\varepsilon)}(t)$ which are uniform in $\varepsilon \in(0,1]$ and in $L$. From this (4.28) follows. 
(b) $g\left(\mathbf{S}^{2}\right)$ is strictly monotone decreasing, and

$$
\int g\left(\mathbf{S}^{2}\right)|\mathbf{S}|^{\eta} d \mathbf{S}<\infty, \text { for some } \eta>0 .
$$

In this example the verification of (4.28), and hence of the hypotheses of Theorem 4.1, proceeds again by estimating $\frac{\partial}{\partial t} \log z_{L}^{(\varepsilon)}(t)$. For $\varepsilon>0$ and $L$ bounded, it is immediate to verify that

$$
\frac{\partial}{\partial t} \log z_{L}^{(\varepsilon)}(t)=2\left\langle g^{\prime}\left(\mathbf{S}_{0}^{2}+2 t\right)\right\rangle_{L, t}^{(\varepsilon)}<0
$$

with $g^{\prime}(x) \equiv \frac{d}{d x} g(x)$.

Thus it suffices to show that

$$
\lim _{\varepsilon>0} \lim _{L>\mathbb{Z}^{v}}\left\langle g^{\prime}\left(\mathbf{S}_{0}^{2}+2 t\right)\right\rangle_{L, t}^{(\varepsilon)}<0
$$

for some interval of values of $t$ of positive measure. Since $g^{\prime}(x)<0$, for all $x \in[0, \infty)$, (4.33) can fail only if $\left|\mathbf{S}_{0}\right|=\infty$, almost surely, in the limit $L \nearrow \mathbb{Z}^{v}, \varepsilon \searrow 0$. This possibility can be excluded if

$$
\lim _{\varepsilon \rtimes 0} \lim _{L>\mathbb{Z}^{v}}\left\langle\left|\mathbf{S}_{0}\right|^{\eta}\right\rangle_{L, t}^{(\varepsilon)} \leqq C_{t},
$$

for some $\eta>0$ and some constant $C_{t}$ which is finite for all $t<\infty$. Using the chessboard estimate [12] we find

$$
\left\langle\left|\mathbf{S}_{0}\right|^{\eta}\right\rangle_{L, t}^{(\varepsilon)}=\left[\int^{-\beta H^{(\varepsilon)}} \prod_{j} g\left(\mathbf{S}_{j}^{2}+2 t\right)\left|\mathbf{S}_{j}\right|^{\eta} d \mathbf{S}_{j} / Z_{L, t}^{(\varepsilon)}\right]^{1 /|L|}
$$

By definition of $H^{(\varepsilon)}$, see (4.3) and (4.4),

$$
0<H^{(\varepsilon)} \leqq 1 / 2(4 d+\varepsilon)\left(\sum_{j \in L}\left|\mathbf{S}_{j}\right|^{2}\right)
$$

Thus

$$
\left\langle\left|\mathbf{S}_{0}\right|^{\eta}\right\rangle_{L, t}^{(\varepsilon)} \leqq \int g\left(\mathbf{S}^{2}+2 t\right)|\mathbf{S}|^{\eta} d \mathbf{S}\left(\int g\left(\mathbf{S}^{2}+2 t\right) e^{-\frac{\beta}{2}(4 d+\varepsilon) \mathbf{S}^{2}} d \mathbf{S}\right)-1
$$

By (4.32), the right side of (4.35) is finite for all $t<\infty$, with a bound which is uniform in $L$ and $\varepsilon$. This yields (4.28).

Remark. In dimension $v \geqq 3$ condition (4.32) is superfluous, i.e. (4.28) holds for every strictly monotone decreasing $g$. For, by (4.24a) and (4.24b) through (4.26)

$$
\left\langle\left|\mathbf{S}_{0}\right|^{2}\right\rangle_{L, t}^{(\varepsilon)} \leqq \frac{1}{\beta}\left(-\Delta_{L}+\varepsilon\right)_{00}^{-1}
$$

hence

$$
\lim _{\varepsilon>0} \lim _{L \rightarrow \mathbb{Z}^{v}}\left\langle\left|\mathbf{S}_{0}\right|^{2}\right\rangle_{L, t}^{(\varepsilon)} \leqq \frac{1}{\beta}(-\Delta)_{00}^{-1}
$$

which implies (4.34) with $\eta=2$ and $C_{t} \leqq C<\infty$ independent of $t$, provided $\nu \geqq 3$. 
(c) $v \geqq 3 ; g$ a monotone decreasing function, with

$$
0<g_{\infty} \equiv \lim _{|\mathbf{S}| \rightarrow \infty} g\left(\mathbf{S}^{2}\right)<g(0) \equiv g_{0}<\infty
$$

As in examples (a) and (b) we must verify (4.28): Given $t$, let

and

$$
g_{\tau}\left(\mathbf{S}^{2}\right)=(1-\tau) g\left(\mathbf{S}^{2}\right)+\tau g\left(\mathbf{S}^{2}+2 t\right)
$$

Then

$$
Z_{L}^{(\varepsilon)}(\tau) \equiv \int e^{-\beta H^{(\varepsilon)}} \prod_{j \in L} g_{\tau}\left(\mathbf{S}_{j}^{2}\right) d \mathbf{S}_{j}
$$

$$
\begin{aligned}
\log z_{L}^{(\varepsilon)}(t) & =\int_{0}^{1} d \tau \frac{\partial}{\partial t} \log Z_{L}^{(\varepsilon)}(\tau) \\
& =\int_{0}^{1}\left\langle g\left(\mathbf{S}_{0}^{2}+2 t\right)-g\left(\mathbf{S}_{0}^{2}\right)\right\rangle_{L}^{(\varepsilon)}(\tau) d \tau,
\end{aligned}
$$

where $\langle-\rangle_{L}^{(\varepsilon)}(\tau)$ is the finite volume expectation defined in (4.6) with $g$ replaced by $g_{\tau}$. By (4.37) we have, for $t>0$,

$$
g\left(\mathbf{S}_{0}^{2}+2 t\right)-g\left(\mathbf{S}_{0}^{2}\right)<-\delta,
$$

if $\mathscr{R}_{1} \leqq\left|\mathbf{S}_{0}\right| \leqq \mathscr{R}_{2}$, for some positive constants $\delta$ and $\mathscr{R}_{1}<\mathscr{R}_{2}$, (depending on $t$ ). We define

Then

$$
X(\mathbf{S})= \begin{cases}1, & \mathscr{R}_{1} \leqq\left|\mathbf{S}_{0}\right| \leqq \mathscr{R}_{2} \\ 0, & \text { otherwise }\end{cases}
$$

$$
\left\langle g\left(\mathbf{S}_{0}^{2}+2 t\right)-g\left(\mathbf{S}_{0}^{2}\right)\right\rangle_{L}^{(\varepsilon)}(\tau) \leqq-\delta\left\langle X\left(\mathbf{S}_{0}\right)\right\rangle_{L}^{(\varepsilon)}(\tau) .
$$

Therefore the proof of (4.28), as $L \nearrow \mathbb{Z}^{v}$ and $\varepsilon \searrow 0$, is complete if we can prove that

$$
\left\langle X\left(\mathbf{S}_{0}\right)\right\rangle_{L}^{(\varepsilon)}(\tau) \geqq C>0,
$$

for $L$ large, $\varepsilon$ small and $t$ large enough. We now claim that if conversely

then

$$
\left.\begin{array}{r}
\left\langle X\left(\mathbf{S}_{0}\right)\right\rangle_{L}^{(\varepsilon)}(\tau)=0, \\
\left\langle X\left(\mathbf{S}_{0}-\mathbf{a}\right)\right\rangle_{L}^{(\varepsilon)}(\tau)=0,
\end{array}\right\}
$$

for all $\mathbf{a} \in \mathbb{R}^{N}$, for all $\varepsilon \geqq 0, L \leqq \mathbb{Z}^{v}$. Consequently, $\mathbf{S}_{0}=\infty$, almost surely. However, in $v \geqq 3$ dimensions,

$$
\lim _{\varepsilon \rtimes 0} \lim _{L>\mathbb{Z}^{\nu}}\left\langle\mathbf{S}_{0}^{2}\right\rangle_{L}^{(\varepsilon)}(\tau) \leqq \frac{1}{\beta}(-\Delta)_{00}^{-1}<\infty,
$$

i.e. $\mathbf{S}_{0}$ is finite, almost surely, when $\varepsilon>0$ and $L$ is large enough. Therefore

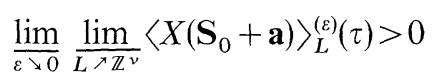

for some $\mathbf{a} \in \mathbb{R}^{N}$ and, by (4.43),

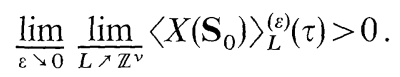


From this we conclude that

for $t>0$. By (4.25) and (4.26)

$$
\varlimsup_{\varepsilon \rtimes 0} \varlimsup_{L>\mathbb{Z}^{v}} z_{L}^{(\varepsilon)}(t)<1,
$$

$$
\lim _{\varepsilon \rtimes 0} \lim _{L>\mathbb{Z}^{\nu}}\left\langle S_{i}^{(\alpha)} S_{j}^{(\alpha)}\right\rangle_{L}^{(\varepsilon)} \leqq \frac{1}{\beta}(-\Delta)_{i j}^{-1} z^{|i-j|},
$$

with

$$
z \leqq \int_{0}^{\infty} e^{-t} \varlimsup_{\varepsilon>0} \varlimsup_{L>\infty} z_{L}^{(\varepsilon)}(t / \beta(2 v+\varepsilon)) d t<1 .
$$

Thus, we are left with proving (4.43). We start by noticing that the measures

$$
\left(Z_{L}^{(\varepsilon)}(\tau)\right)^{-1} e^{-\beta H^{(\varepsilon)}} \prod_{j \in L} g_{\tau}\left(\mathbf{S}_{j}^{2}\right) d S_{j}
$$

are quasi-invariant under the substitution

$$
\mathbf{S}_{0} \rightarrow \mathbf{S}_{0}+\mathbf{a}, \mathbf{a} \in \mathbb{R}^{N},
$$

with a Radon-Nikodym derivative, $\varrho_{\mathbf{a}}(\mathbf{S})$, given by

$$
\varrho_{\mathbf{a}}(\mathbf{S})=e^{-\frac{\beta}{2}(2 v+\varepsilon) \mathbf{a}^{2}} e^{-\beta \varepsilon\left(\mathbf{S}_{0} \cdot \mathbf{a}\right)} \exp \left[\beta \sum_{|k|=1}\left(\mathbf{S}_{k}-\mathbf{S}_{0}\right) \cdot \mathbf{a}\right] \frac{g_{\tau}\left(\left(\mathbf{S}_{0}+\mathbf{a}\right)^{2}\right)}{g_{\tau}\left(\mathbf{S}_{0}^{2}\right)} .
$$

We now show that for all $p<\infty$

$$
\left\langle\varrho_{\mathbf{a}}(\mathbf{S})^{p}\right\rangle_{L}^{(\varepsilon)}(\tau)<C_{p},
$$

for some finite constant $C_{p}$ independent of $\varepsilon$ and $L$. First, we note that

$$
\left(g_{\tau}\left(\left(\mathbf{S}_{0}+\mathbf{a}\right)^{2}\right) / g_{\tau}\left(\mathbf{S}_{0}^{2}\right)\right)^{3 p} \leqq\left(g_{0} / g_{\infty}\right)^{3 p}<\infty,
$$

for all $p<\infty$ and all $\tau$ and $t$. Second,

$$
\left\langle\exp \left[3 p \beta \sum_{|k|=1}\left(\mathbf{S}_{k}-\mathbf{S}_{0}\right) \cdot \mathbf{a}\right]\right)_{L}^{(\varepsilon)}(\tau) \leqq \exp \left(9 p^{2} \beta \mathbf{a}^{2} / 2\right),
$$

by Gaussian domination [13]. Finally, using the chessboard estimate [12]

$$
\left\langle e^{-3 p \beta \varepsilon\left(\mathbf{S}_{0} \cdot \mathbf{a}\right)}\right\rangle_{L}^{(\varepsilon)}(\tau) \leqq \mathrm{e}^{9 p^{2} \beta \varepsilon|\mathbf{a}|^{2} / 2}\left[Z_{L}^{(\varepsilon)}(\tau, \mathbf{a}) / Z_{L}^{(\varepsilon)}(\tau)\right]^{1 /|L|},
$$

where $Z_{L}^{(\varepsilon)}(\tau, \mathbf{a})$ is given by (4.38), but with $g_{\tau}\left(\mathbf{S}_{j}^{2}\right)$ replaced by $g_{\tau}\left(\left(\mathbf{S}_{j}^{2}+3 p \mathbf{a}\right)^{2}\right)$, for all $j$. [To prove (4.46) one first applies the chessboard estimate and then changes variables, $\mathbf{S}_{j} \rightarrow \mathbf{S}_{j}+3 p \mathbf{a}, j \in L$, using the invariance of $\left(\mathbf{S}, \Delta_{L} \mathbf{S}\right)$ under that change of variables.] It is easy to see that the second factor on the right side of (4.46) is bounded by $g_{0} / g_{\infty}$. This completes our proof of (4.45). In order to prove (4.43), we note that

$$
\begin{aligned}
\left\langle X\left(\mathbf{S}_{0}-\mathbf{a}\right)\right\rangle_{L}^{(\varepsilon)}(\tau) & =\left\langle X\left(\mathbf{S}_{0}\right) \varrho_{\mathbf{a}}(\mathbf{S})\right\rangle_{L}^{(\varepsilon)}(\tau) \\
& \leqq\left(\left\langle X\left(\mathbf{S}_{0}\right)\right\rangle_{L}^{(\varepsilon)}(\tau)\right)^{1 / q}\left(\left\langle\varrho_{\mathbf{a}}(\mathbf{S})^{p}\right\rangle_{L}^{(\varepsilon)}(\tau)\right)^{1 / p} \\
& \leqq C_{p}^{1 / p}\left(\left\langle X\left(\mathbf{S}_{0}\right)\right\rangle_{L}^{(\varepsilon)}(\tau)\right)^{1 / q}
\end{aligned}
$$

with $1 / p+1 / q=1$, and we have used (4.45).

This completes the proof of (4.28) for example (c). 


\section{Gaussian Inequalities}

The Lebowitz inequality [6], originally proven for Ising models in zero magnetic field, says

$$
\begin{aligned}
\left\langle S_{i} S_{j} S_{k} S_{l}\right\rangle \leqq & \left\langle S_{i} S_{j}\right\rangle\left\langle S_{k} S_{l}\right\rangle \\
& +\left\langle S_{i} S_{k}\right\rangle\left\langle S_{j} S_{l}\right\rangle+\left\langle S_{i} S_{l}\right\rangle\left\langle S_{j} S_{k}\right\rangle .
\end{aligned}
$$

In [7], Newman generalized these inequalities to

$$
\left\langle S_{i} F\right\rangle \leqq \sum_{j}\left\langle S_{i} S_{j}\right\rangle\left\langle\frac{\partial F}{\partial S_{j}}\right\rangle,
$$

where $F$ is a polynomial with positive coefficients. [(5.1) follows from (5.2) by taking $F$ to be a product of three spins.] He gave (5.2) the name "Gaussian Domination" because the inequality would be saturated if $\langle\cdot\rangle$ were gaussian.

The inequality, (5.1), has been extended, [16], to models of the form (2.3) with $N=1$ components and

$$
g_{i}\left(S_{i}^{2}\right)=e^{-V\left(S_{\imath}\right)},
$$

where $V$ is even and $V^{\prime \prime \prime} \geqq 0$ on the positive real axis. Results for $N=2,3,4$ components also exist [17].

We shall rederive and extend these results for $N=1,2$. Our class of models has single spin distributions of the form

$$
g_{i}\left(S_{i}^{2}\right)=e^{-f_{\imath}\left(S_{l}^{2}\right)}
$$

with $f^{\prime \prime}(t) \geqq 0$ for $t \in[0, \infty)$. This class of single spin distributions overlaps with but is not identical to the Ellis, Monroe, Newman class (5.3). Many well-known models such as Ising models and $\phi^{4}$ field theories are in both. Some good features of our proof are that we obtain the stronger version of gaussian domination (5.2), and our method is simple and suggests many variations on the same theme. We will explore one of these in the next section.

We shall demonstrate that these inequalities are really a consequence of Griffiths II inequalities. For $N=2$ components, the analogue of these are the Ginibre inequalities, [18], and so our method will also produce gaussian domination results for $N=2$. The analogous Griffiths-Ginibre type inequalities are expected but not known to hold for $N>2$, so this is the obstacle to extending our results to $N>2$.

Let $F$ be a polynomial in $\underset{\sim}{S}$ with positive coefficients. By Theorem 2.2

$$
\left.\left\langle S_{i} F\right\rangle=\sum_{j} \sum_{\omega: i \rightarrow j} J_{\omega} \int d v_{\omega}(t)\left[\frac{\partial F}{\partial S_{j}}\right]_{t}\right] / Z
$$

(the $Z$ normalizes $[-]$ to $\langle-\rangle$ ). Furthermore

$$
\frac{1}{Z}\left[\frac{\partial F}{\partial S_{j}}\right]_{\underline{t}}=\frac{Z_{t}}{Z}\left\langle\frac{\partial F}{\partial S_{j}}\right\rangle_{\underline{\tau}}
$$


(Recall that $Z_{t} \equiv[1]_{t}$.) By the Griffiths II inequality as stated in $[5, \mathrm{p} .120]$

$$
\left\langle\frac{\partial F}{\partial S_{j}}\right\rangle_{t} \text { is decreasing in } \stackrel{t}{\sim}
$$

as can be seen by differentiating with respect to $t_{i} \in t$. Therefore

$$
\frac{1}{Z}\left[\frac{\partial F}{\left.\partial S_{j}\right]_{t}}\right]_{\leqq} \frac{Z_{t}}{Z}\left\langle\frac{\partial F}{\partial S_{j}}\right\rangle_{t=0}=\frac{Z_{t}}{Z}\left\langle\frac{\partial F}{\partial S_{j}}\right\rangle
$$

and so

$$
\left\langle S_{i} F\right\rangle \leqq \sum_{j}\left\{\sum_{\omega: i \rightarrow j} J_{\omega} \int d v_{\omega}(t) \frac{Z_{t}}{Z}\right\} \cdot\left\langle\frac{\partial F}{\partial S_{j}}\right\rangle .
$$

By taking $F=S_{j}$ in (5.5) we see that the quantity in curly brackets is $\left\langle S_{i} S_{j}\right\rangle$ and so (5.6) becomes (5.2). In this way we obtain the following gaussian domination result :

Theorem 5.1. If $\langle(\cdot)\rangle$ is ferromagnetic $\left(J_{i j} \geqq 0\right)$ and has single spin distributions satisfying (2.5) and

$$
g\left(S^{2}\right)=e^{-f\left(S^{2}\right)}
$$

with $f^{\prime \prime}(t) \geqq 0$ on $[0, \infty)$, then

$$
\left\langle S_{i} F\right\rangle \leqq \sum_{j}\left\langle S_{i} S_{j}\right\rangle\left\langle\frac{\partial F}{\partial S_{j}}\right\rangle,
$$

where $F$ can be any function of $\underset{\sim}{S}$ of the form

$$
F=\prod_{i} F_{i}\left(S_{i}\right)
$$

with each $F_{i}$ being either odd or even and $F_{i}^{\prime}(t), F_{i}^{\prime \prime}(t) \geqq 0$ on $[0, \infty)$.

We can allow $F$ to have this more general form because the Griffiths II inequalities hold for this class of functions.

Remark. For $N=2$ the same methods show that for $\alpha=1,2$

$$
\left\langle S_{i}^{(\alpha)} F\right\rangle \leqq \sum_{j}\left\langle S_{i}^{(\alpha)} S_{j}^{(\alpha)}\right\rangle\left\langle\frac{\partial F}{\partial S_{j}^{(\alpha)}}\right\rangle,
$$

provided $F$ is a polynomial in $S^{(\alpha)}$ with positive coefficients and $f$ is a polynomial with positive coefficients for terms of degree greater than 1.

\section{Application to the Lieb-Rivasseau Improvement of Simon's Inequality}

We consider the same class of models as in the last section:

$$
\begin{aligned}
\langle-\rangle & \equiv[-] /[1], \\
{[-] } & \equiv \int \prod_{i \in L} e^{-f_{i}\left(\mathbf{S}_{i}^{2}\right)} d \mathbf{S}_{i} e^{-H}-, \\
H & \equiv-\frac{1}{2} \sum_{\substack{i, j \in L \\
\alpha}} S_{i}^{(\alpha)} J_{i j} j_{j}^{(\alpha)} ; \quad J_{i j} \geqq 0,
\end{aligned}
$$


and we require that $\exp \left(-f_{i}\right)$ obey $(2.5)$ and

$$
f_{i}^{\prime \prime}(t) \geqq 0 \quad \text { if } \quad t \geqq 0 \quad(N=1)
$$

for 1-component models, and for two-component models $f_{i}$ must be a polynomial whose coefficients for degree greater than 1 are positive. Furthermore, as in the last section, the limitation $N=1,2$ comes from not knowing the Griffiths II respectively Ginibre - inequalities if $N>2$.

The inequality we are about to state and prove was, aside from a generalization we have made, obtained by Simon [8] and improved by Lieb [9] and Rivasseau [10]. The proof Simon gave was based on the Lebowitz inequality (see Sect. 5). We have simply noted that the gaussian domination result we have stated in the last section is sufficiently powerful to quickly reproduce the improved inequality for $N=1$ and 2 by a proof closely resembling Simon's proof.

Define, for $\Omega$ any subset of $L$,

$$
H_{\Omega} \equiv-\frac{1}{2} \sum_{\substack{i, j \in \Omega \\ \alpha}} S_{i}^{(\alpha)} J_{i j} S_{j}^{(\alpha)}
$$

Corresponding to $\langle-\rangle$ we have $\langle-\rangle_{\Omega}$ which is obtained by replacing $L$ by $\Omega$ throughout all definitions.

Theorem 6.1. Let $i$ be a site in $L$ and let $\Omega \subset L$ contain $i$. Suppose $F$ is a polynomial in the $\alpha^{\text {th }}$ component of the spins $\mathbf{S}_{j}$ which is independent of the spins in $\Omega$, then

$$
\left\langle S_{i}^{(\alpha)} F\right\rangle \leqq \sum_{\substack{j \in \Omega \\ k \notin \Omega}}\left\langle S_{i}^{(\alpha)} S_{j}^{(\alpha)}\right\rangle_{\Omega} J_{j k}\left\langle S_{k}^{(\alpha)} F\right\rangle .
$$

Proof. We define a new Hamiltonian $H^{\partial \Omega}$ by setting to zero all $J_{i j}$ coefficients for which $i \in \Omega, j \notin \Omega$, i.e.

$$
H^{\partial \Omega} \equiv H_{\Omega}+H_{L \sim \Omega}
$$

and we define a corresponding expectation $\langle-\rangle^{\partial \Omega}$. In terms of this we can write the standard expectation as follows

$$
\begin{gathered}
\langle-\rangle=\langle-\Phi\rangle^{\partial \partial \Omega} /\langle\Phi\rangle^{\partial \Omega}, \\
\Phi \equiv \exp \left(\frac{1}{2} \sum_{\substack{i \in \Omega \\
j \notin \Omega \\
\alpha}} S_{i}^{(\alpha)} J_{i j} S_{j}^{(\alpha)}\right) .
\end{gathered}
$$

By the gaussian domination result, Theorem 3.1, with $F$ replaced by $F \Phi$, we obtain (leaving off $\alpha$ superscripts)

$$
\begin{aligned}
\left\langle S_{i} F\right\rangle & =\left\langle S_{i} F \Phi\right\rangle^{\partial \Omega} /\langle\Phi\rangle^{\partial \Omega} \\
& \leqq \sum_{j}\left\langle S_{i} S_{j}\right\rangle^{\partial \Omega}\left\langle\frac{\partial}{\partial S_{j}}(F \Phi)\right\rangle^{\partial \Omega}\left\langle\langle\Phi\rangle^{\partial \Omega}\right.
\end{aligned}
$$


Now use

$$
\begin{aligned}
\left\langle\frac{\partial}{\partial S_{j}}(F \Phi)\right\rangle^{\partial \Omega} & =\left\langle F \frac{\partial \Phi}{\partial S_{j}}\right\rangle^{\partial \Omega} \\
& =\sum_{\substack{j \in \Omega \\
k \notin \Omega}} J_{j k}\left\langle F S_{k} \Phi\right\rangle^{\partial \Omega},
\end{aligned}
$$

and note in the result that by (6.2)

$$
\left\langle F S_{k} \Phi\right\rangle^{\partial \Omega} /\langle\Phi\rangle^{\partial \Omega}=\left\langle F S_{k}\right\rangle ; \quad\left\langle S_{i} S_{j}\right\rangle^{\partial \Omega}=\left\langle S_{i} S_{j}\right\rangle_{\Omega} .
$$

Theorem 6.1 is proved.

Theorem 5.1 applies in the above proof because $\Phi$ can be approximated by a polynomial with positive coefficients.

\section{The Mass Gap for $\left(\phi^{4}\right)_{2}$}

The proof of the mass gap for weakly coupled $\lambda P(\phi)_{2}$ models was first established by a cluster expansion [19]. In this section we shall establish the mass gap for $\lambda\left(\phi^{4}\right)_{2}$ using integration by parts (2.17) and Theorem 6.1. The proof is simple, moreover one can establish reasonably good values of $\lambda$ for which the mass gap occurs. On the other hand our method does not apply to higher degree polynomials, nor does it yield analyticity in the coupling.

We first consider the $\lambda \phi^{4}$ model on a lattice $\delta \mathbb{Z}^{2} \subset \mathbb{R}^{2}$. The lattice spacing $\delta$ will later be sent to zero. For notational purposes we set $N=1$. Let $\Lambda$ be a large rectangle containing $(0,0)$. We define

$$
H_{\Lambda}(\phi)=-\frac{1}{2} \sum_{|i-j|=\delta} \phi(i) \phi(j), \quad i, j \in \Lambda \cap \delta \mathbb{Z}^{2}
$$

and

$$
g_{\delta}\left(\phi^{2}(j)\right)=\exp \left\{-2 \phi^{2}(j)-\delta^{2}\left[\phi^{2}(j)+\lambda: \phi^{4}(j):_{\delta}\right]\right\} .
$$

Thus $J_{i j}=1$ if $|i-j|=\delta$ and $i, j \in \Lambda$ and $J_{i j}=0$ otherwise. The Wick order for $: \phi^{4}:_{\delta}$ is defined with respect to $G^{\delta}(x-y)$, the Green's function of $-\Delta+1$ on the lattice. The Fourier transform of $G^{\delta}$ is given by

$$
\hat{G}^{\delta}(p)=\left\{2 \delta^{-2}\left[2-\cos \left(\delta p_{1}\right)-\cos \left(\delta p_{2}\right)\right]+1\right\}^{-1} .
$$

In the continuum limit $\delta \rightarrow 0, \hat{G}(p)=\left(p^{2}+1\right)^{-1}$ which is the Euclidean free field propagator. The normalized correlations are defined by

$$
\langle\phi(0) \phi(x)\rangle_{\Lambda}(\delta, \lambda)=\frac{\int \phi(0) \phi(x) e^{-H_{\Lambda}(\phi)} \prod_{j \in \Lambda} g_{\delta}\left(\phi^{2}(j)\right) d \phi(j)}{\int e^{-H_{\Lambda}(\phi)} \prod_{j \in \Lambda} g_{\delta}\left(\phi^{2}(j)\right) d \phi(j)} .
$$

In the continuum limit this expectation is

$$
\frac{\int \phi(0) \phi(x) e^{-\lambda V(\phi, \Lambda)} d \mu_{\Lambda}(\phi)}{\int e^{-\lambda V(\phi, \Lambda)} d \mu_{\Lambda}(\phi)} .
$$


Here $d \mu_{\Lambda}$ is the Gaussian measure of mean 0 and covariance $\left(-\Delta_{\Lambda}+1\right)^{-1}$ and $V(\phi, \Lambda)=\int_{\Lambda}: \phi^{4}(x): d x$. The subscript $\Lambda$ on $\Delta$ means that 0 Dirichlet boundary conditions are imposed at $\partial \Lambda$. We now state the main theorem of this section.

Theorem 7.1. Let \langle\rangle$_{\Lambda}(\delta, \lambda)$ be defined as above. There are constants $\lambda_{0}>0, m>0$, $C$ independent of $\Lambda$ such that

$$
\lim _{\delta \downarrow 0}\langle\phi(0) \phi(x)\rangle_{\Lambda}(\delta, \lambda) \leqq C e^{-m|x|}
$$

whenever $0 \leqq \lambda \leqq \lambda_{0},|x| \geqq 1$.

Remarks. Our proof also applies to the two-component $\left(\phi^{4}\right)_{2}$ model. The exponential clustering of the $n$ point correlations follows from (7.2) and the gaussian inequalities. Hence we have established a mass gap.

Proof. Let $\Omega \subset \Lambda$ be a square centered at the origin with sides of length $l$. By Theorem 6.1

$$
\langle\phi(0) \phi(x)\rangle_{\Lambda}(\delta, \lambda) \leqq \sum\langle\phi(0) \phi(z)\rangle_{\Omega}(\delta, \lambda)\left\langle\phi\left(z^{\prime}\right) \phi(x)\right\rangle_{\Lambda}(\delta, \lambda),
$$

where the sum ranges over $z \in \Omega, z^{\prime} \notin \Omega$ and $\left|z-z^{\prime}\right|=\delta$. We shall show that for $0 \leqq \lambda \leqq \lambda_{0}$ and some $l \gg 1$,

$$
\sum_{z \in \partial \Omega}\langle\phi(0) \phi(z)\rangle_{\Omega}(\delta, \lambda) \leqq \gamma<1
$$

where $\lambda_{0}, l$, and $\gamma$ are independent of $\delta$. As in [8] successive iterations of (7.3) and (7.4) imply exponential decay of the two point function.

In order to prove (7.4) let $z \in \partial \Omega$, then the integration by parts formula shows that

$$
\begin{aligned}
\delta^{-1}\langle\phi(0) \phi(z)\rangle_{\Omega}(\delta, \lambda)= & \delta^{-1} G_{\Omega}^{\delta}(0, z) \\
& -4 \lambda \delta^{2} \sum_{y \in \Omega}\left\langle\phi(0): \phi^{3}(y):_{\delta}\right\rangle \delta^{-1} G_{\Omega}^{\delta}(y, z)
\end{aligned}
$$

Now it suffices to show that (7.5) is less than $[4 l]^{-1}$ for any $z \in \partial \Omega$. Suppose that $z=\left(\frac{l}{2}, z_{2}\right)$. Let $G_{l}^{\delta}(x, y)$ be the Green's function with zero Dirichlet boundary conditions on the line $x_{1}=\frac{l}{2}+\delta$. By the random path expansion, Theorem 1.1, and the reflection principle we know that

$$
0 \leqq G_{\Omega}^{\delta}(y, z) \leqq G_{l}^{\delta}(y, z)=G^{\delta}(y-z)-G^{\delta}(y-\tilde{z}),
$$

where $\tilde{z}=\left(\frac{l}{2}+2 \delta, z_{2}\right)$ is the image of $z$. Hence

$$
\delta^{-1} G_{l}^{\delta}(y, z)=\frac{2}{4 \pi^{2}} \int_{\left|p_{\imath}\right| \leqq \delta^{-1} \pi} e^{i p(y-z)} e^{-i p_{1} \cdot \delta}\left[\frac{\sin p_{1} \delta}{\delta}\right] \hat{G}^{\delta}(p) d p_{1} d p_{2} .
$$


It is not difficult to show that $\delta^{-1} G_{l}^{\delta}(y, z)$ approaches $\frac{\partial}{\partial z_{1}} G(x, z)$ as $\delta \rightarrow 0$. Moreover

$$
\begin{array}{rlrl}
\delta^{-1} G_{l}^{\delta}(y, z) & \leqq K e^{-1 / 2|y-z|} & \text { if } & |y-z| \geqq 1 \\
\leqq K|y-z|^{-1} & \text { if } & |y-z| \leqq 1
\end{array}
$$

where $K$ is independent of $\delta$ and $l$. These bounds may be checked by explicitly calculating the $d p_{1}$ integral using a contour integral.

The above inequalities show that the first term on the right side of (7.5) is bounded by $\left(e^{-l / 2}\right)$. To estimate the second term we again integrate by parts and obtain

$$
12 \lambda \delta^{2} \sum_{y \in \Omega}\left\langle: \phi^{2}(y):_{\delta}\right\rangle_{\Omega}(\delta, \lambda) G_{\Omega}^{\delta}(0, y) \delta^{-1} G_{\Omega}^{\delta}(y, z)-16 \lambda^{2}\left\langle F_{1} F_{2}\right\rangle_{\Omega}(\delta, \lambda)
$$

where

$$
\begin{aligned}
& F_{1}=\delta^{2} \sum_{y^{\prime} \in \Omega}: \phi^{3}\left(y^{\prime}\right):_{\delta} G_{\Omega}^{\delta}\left(0, y^{\prime}\right) \\
& F_{2}=\delta^{2} \sum_{y \in \Omega}: \phi^{3}(y):_{\delta} \delta^{-1} G_{\Omega}^{\delta}(y, z) .
\end{aligned}
$$

The first term on the right side of (7.7) is small as $\lambda \rightarrow 0$ since $\left\langle: \phi^{2}(y):\right\rangle_{\delta}(\delta, \lambda)$ is uniformly bounded as $\delta \downarrow 0$. To bound the second term we apply the Schwarz inequality with respect to the Gaussian measure. Thus

$$
\left\langle F_{1} F_{2}\right\rangle_{\Omega}(\delta, \lambda) \leqq\left\langle\left(F_{1} F_{2}\right)^{2}\right\rangle_{\Omega}^{1 / 2}(\delta, \lambda=0) \cdot\left\langle e^{-2 \lambda V(\phi, \Omega)}\right\rangle_{\Omega}^{1 / 2}(\delta, 0) \cdot Z^{-1}
$$

The right side is bounded, using standard estimates, by const exp[ $\lambda$ const $\left.l^{2}\right]$ for $0 \leqq \lambda \leqq 1$. The partition function $Z$ is bounded from below using Jensen's inequality. Hence we can fix $l$ large and $\lambda_{0}(l)$ small so that $\gamma$ in (7.4) is less than 1. End of proof of Theorem 7.1.

\section{References}

1. Symanzik, K. : Euclidean quantum field theory. In : Local quantum theory. Jost, R. (ed.) New York, London: Academic Press 1969

2. Brydges, D., Federbush, P.: Commun. Math. Phys. 62, 79 (1978) (also in unpublished work by J. Fröhlich and T. Spencer)

3. Durhuus, B., Fröhlich, J.: Commun. Math. Phys. 75, 103 (1980)

4. Fröhlich, J., Israel, R., Lieb, E.H., Simon, B.: Commun. Math. Phys. 62, 1 (1978)

5. Simon, B.: Functional integration and quantum physics. New York: Academic Press 1979

6. Lebowitz, J.L.: Commun. Math. Phys. 28, 313 (1972). See also [5] p. 131

7. Newman, C.: Z. Warsch. verw. Gebiete 33, 75 (1975)

8. Simon, B.: Commun. Math. Phys. 77, 111 (1980)

9. Lieb, E.H.: Commun. Math. Phys. 77, 127 (1980)

10. Rivasseau, V.: Commun. Math. Phys. 77, 145 (1980)

11. Aizenman, M., Simon, B.: Commun. Math. Phys. 77, 137 (1980)

12. Glimm, J., Jaffe, A., Spencer, T.: Commun. Math. Phys. 45, 203 (1975)

Fröhlich, J., Lieb, E.H.: Commun. Math. Phys. 60, 233 (1978)

Seiler, E., Simon, B.: Ann. Phys. (NY) 97, 470 (1976) 
13. Fröhlich, J., Simon, B., Spencer, T.: Commun. Math. Phys. 50, 79 (1976)

14. Lebowitz, J.L.: Commun. Math. Phys. 28, 313 (1972)

15. McBryan, O., Spencer, T.: Commun. Math. Phys. 53, 299 (1977)

16. Ellis, R.S., Monroe, J.L., Newman, C.M. : Commun. Math. Phys. 46, 167 (1976)

17. Bricmont, J.: J. Stat. Phys. 17, 289 (1977)

18. Ginibre, J.: Commun. Math. Phys. 16, 310 (1970)

19. Glimm, J., Jaffe, A., Spencer, T.: Ann. Math. 100, 585 (1974)

Glimm, J., Jaffe, A., Spencer, T.: The particle structure of the weakly coupled $P(\phi)_{2}$ model and other applications of high temperature expansions. In: Constructive quantum field theory, Velo, G., Wightman, A.S. (eds.), p. 133. Berlin, Heidelberg, New York: Springer 1973

Communicated by A. Jaffe

Received September 24, 1981 